\title{
Bioengineered Livers: A New Tool for Drug Testing and a Promising Solution to Meet the Growing Demand for Donor Organs
}

\author{
Franziska Mußbach $^{a}$ Utz Settmacher ${ }^{b}$ Olaf Dirsch $^{c} \quad$ Chichi Xie $^{a}$ \\ Uta Dahmen a \\ a Experimental Transplantation Surgery, Department of General, Visceral and Vascular \\ Surgery, University Hospital Jena, and ${ }^{\mathrm{b}}$ Department of General, Visceral and Vascular \\ Surgery, University Hospital Jena, Jena, and ' Institute of Pathology, Hospital Chemnitz \\ gGmbH, Chemnitz, Germany
}

\section{Key Words}

Decellularization · Liver engineering · Organ engineering · Organ shortage $\cdot$ Repopulation

\begin{abstract}
Background: Organ engineering is a new innovative strategy to cope with two problems: the need for physiological models for pharmacological research and donor organs for transplantation. A functional scaffold is generated from explanted organs by removing all cells (decellularization) by perfusing the organ with ionic or nonionic detergents via the vascular system. Subsequently the acellular scaffold is reseeded with organ-specific cells (repopulation) to generate a functional organ. Summary: This review gives an overview of the state of the art describing the decellularization process, the subsequent quality assessment, the repopulation techniques and the functional assessment. It emphasizes the use of scaffolds as matrix for culturing human liver cells for drug testing. Further, it highlights the techniques for transplanting these engineered scaffolds in allogeneic or xenogeneic animals in order to test their biocompatibility and use as organ grafts. Key Messages: The first issue is the so-called decellularization, which is best explored and resulted in a multitude of different protocols. The most promising approach seems to be the combination of pulsatile perfusion of the liver with Triton X-100 and SDS via hepatic artery and portal vein. Widely accepted parameters of quality control include the quantitative assessment of the DNA content and the visualization of eventually remaining nuclei confirmed by HE staining. Investigations regarding the composition of the extracellular matrix focused on histological determination of laminin, collagen, fibronectin and elastin and remained qualitatively. Repopulation is the second issue which is addressed. Selection of the most suitable cell type is a highly controversial topic. Currently, the highest potential is seen for progenitor and stem cells. Cells are infused into the scaffold and cultured
\end{abstract}


Mußbach et al.: Bioengineered Livers: A New Tool for Drug Testing and a Promising

under static conditions or in a bioreactor allowing dynamic perfusion of the scaffold. The quality of repopulation is mainly assessed by routine histology and basic functional assays. These promising results prompted to consider the use of a liver scaffold repopulated with human cells for pharmacological research. Transplantation of the (repopulated) scaffold is the third topic which is not yet widely addressed. Few studies report the heterotopic transplantation of repopulated liver tissue without vascular anastomosis. Even fewer studies deal with the heterotopic transplantation of a scaffold or a repopulated liver lobe. However, observation time was still limited to hours, and long-term graft survival has not been reported yet. These exciting results emphasize the potential of this new and promising strategy to create physiological models for pharmacological research and to generate liver grafts for the transplant community to treat organ failure. However, the scientific need for further development in the field of liver engineering is still tremendous.

(C) 2016 S. Karger AG, Basel

\section{Introduction}

Organ engineering is the answer to two seemingly unrelated problems: the need of pharmacological research for more physiological cell culture models and the unmet need of the transplant community for more donor organs.

Organ engineering 'employs aspects of cell biology, material sciences and bioengineering to develop biological substitutes that can restore and maintain the normal function of damaged tissues and organs' [1]. The aim of this experimental field is to generate functional 3-dimensional organ scaffolds for later repopulation with organ-specific cells.

Currently, two strategies for generating organ scaffolds are being investigated: using synthetic polymers and organ-derived biological scaffolds. The matrix forming the scaffold can be constructed using synthetic (polyglycolic acid, polycaprolactone, polylactic acid) or biological (collagen, fibrin, glycosaminoglycans) polymers. Subsequently, this matrix is used for seeding with organ-specific cells and subsequent cultivation. The applied polymers provide the physical support and biological stimulus to ensure the growth and function of the reseeded cells as a prerequisite to function as a surrogate for an organ. However, up to now, this concept is limited to organs with mechanical or structural functions like skin, cartilage or bladder. Organs with a complex structure and function like the liver cannot be reconstructed with the help of synthetic and biological polymers.

Liver engineering using organ-derived biological scaffolds is the strategy addressing the limitations of using synthetic polymers. The aim is to create an organ scaffold from grafts or organs which are not suitable for transplantation. The generation of the scaffold is performed through a process called decellularization by perfusing the organs with ionic or nonionic detergents. This results in a clear colorless scaffold with a preserved vascular structure. Both the intact vascular structure and the complete removal of parenchymal and endothelial cells, are essential for subsequent repopulation. Repopulation is the term describing the reseeding of the organ scaffold with organ-specific cells to obtain a fully functional organ.

This technique was first successfully applied to the heart by the group of Ottand colleagues in 2008, and later the technique was also applied to other organs like kidneys and lungs [1-4]. Based on the promising results using rodents, the research was also extended to different animal species such as pigs and primates. Even human organs were used, as reported in detail in the review of Scarritt et al. [4]. As a next step, this approach was successfully transferred to the more complex liver organ. The key principle of using detergents to perfuse the organ to obtain an acellular scaffold and reseeding with organ-specific cells or stem cells to obtain a functional organ remained the same. 
Mußbach et al.: Bioengineered Livers: A New Tool for Drug Testing and a Promising

Solution to Meet the Growing Demand for Donor Organs

The repopulated livers can potentially serve two different purposes: in the nearer future, they could be used as humanized cell culture models for pharmacological studies and, probably further away, as engineered organ grafts for transplantation.

The pharmacological research is in need of human physiological models because currently applied research models have limitations. These limitations are mostly due to the reduced transferability into clinical use since the experimental testing is carried out in vitro in cell culture or in vivo in animal models. The newly engineered organs may overcome these limitations and provide more physiological conditions to test the effect of novel drugs, their toxicity and related cellular mechanisms.

The transplant community is in need of donor organs to treat end-stage organ failure. Transplant indications are constantly expanded, whereas both organ donation rates and overall organ quality are currently declining leading to a growing demand. Clinical strategies to meet the rising demand consist of living donation, organ splitting, the use of so-called 'marginal' organs and, in some countries, the use of organs after cardiac death (non-heart beating donors). Despite all efforts, the number of available organs does not meet the clinical need. Newly engineered organs using biological scaffolds could potentially overcome this shortage.

This review is dedicated to illustrating the state of the art and the research questions that need to be addressed in biological liver engineering. To generate a biological whole-organ scaffold, three steps must be considered: decellularization to obtain an acellular organ scaffold, repopulation with organ-specific cells and functional testing of the repopulated organ scaffold. The principal goal is to achieve a fully functional organ.

\section{Source of Organs for Liver Engineering}

The first critical choice is the selection of the species used to obtain the organ scaffold. The selection of the species is dependent on the aim of the study.

\section{Previous Experience}

In fundamental studies, rodents such as mice, rats, ferrets and rabbits are used [5-24]. In translational studies, large animals such as pigs and sheep are preferred [25-32]. The use of pigs as organ donors is discussed controversially because of the immunological barrier due to the expression of galactose-1,3-alpha-galactose in porcine organs which may lead to hyperacute rejection of the repopulated organ.

The newest approach also tested the decellularization process using healthy human livers which are rejected for transplantation due to medical reasons (prolonged cold ischemic time of the graft, the presence of extrahepatic malignancy or other important extrahepatic comorbidities in donors or recipients) [33].

\section{Research Need}

Further research is needed with respect to the appropriate organ source. The use of xenogeneic organs calls for an ethical discussion in addition to the scientific issues. In the case of using a human organ, complex questions need to be addressed. Using organs not suitable for transplantation because of other medical reasons or due to the underlying parenchymal pathology (e.g. severe steatosis) would further extend the donor pool. In 2014, more than 850 brain-dead donors were listed, but 80 of them were rejected as donors due to medical reasons [34]. These organs could be potentially used for liver engineering. Criteria need to be defined to clearly distinguish between a marginal organ suitable for transplantation with an acceptable risk for the recipient and an organ not suitable for transplantation but useful for liver engineering. 
Mußbach et al.: Bioengineered Livers: A New Tool for Drug Testing and a Promising Solution to Meet the Growing Demand for Donor Organs

Up to now, the impact of ischemia and cardiac arrest on the decellularization process has not been explored systematically. The number of potential donors would be even further increased if the decellularization procedure was performed immediately after cardiac arrest using non-heart beating donors and even more, when decellularization could take place several hours after cardiac death.

\section{The Decellularization Process}

In contrast to artificially engineered organ scaffolds using polymer materials, the decellularization of a whole organ is an attractive technique to obtain an organ scaffold with the structure and architecture of the original organ including the vascular structure and the native extracellular matrix.

\section{Previous Experience}

To obtain an acellular scaffold, the explanted liver is perfused via the vascular system with ionic or nonionic detergents. Ionic detergents used for the perfusion include SDS (sodium dodecyl sulfate), Na-deoxycholate, highly concentrated sodium chloride solution and peracidic acid. As nonionic detergent, only Triton X-100 is used for perfusion. SDS is suitable for removing the cellular content within the dense organ structure because it dissolves the cytoplasmic and nuclear membranes by disrupting the membrane bilayer. Limitations exist with respect to the fact that SDS may damage the ultrastructure and denature proteins including the membrane-bound growth factors. Triton X-100 removes the cellular material by disrupting the DNA-protein interactions, as well as lipid-lipid, lipid-protein and protein-protein interactions. However, the exclusive application of this detergent resulted in incomplete decellularization with nuclear material remaining in the scaffold. In most previous studies, a combination of these two detergents was applied $[6,8,10,13,14,16,22,24,26,29,35,36]$. As a result, no remaining cellular and nuclear material was detected, and the structure of the extracellular components was maintained (fig. 1).

The direct comparison regarding the effectivity of the decellularization process is rather difficult due to the high variety in the details of the decellularization protocols and methods applied. Most studies reported their results obtained with a single protocol, but did not compare protocols.

The concentrations of the detergents used for organ perfusion ranged from $0.01 \%$ up to $1 \%$ for the SDS solution and $0.5-3 \%$ for Triton X-100 [9, 19, 20, 23, 24, 37]. Other solutions were used in the same concentrations by all authors, e.g. $3.4 \mathrm{M}$ sodium chloride solution, $1 \%$ peracidic acid and $1 \%$ of sodium deoxycholate solution $[7,18,31]$.

The flow rate employed in the protocols ranged from 0.5 to $50 \mathrm{ml} / \mathrm{min}$. The time also ranged from less than $3 \mathrm{~h}$ [22] up to several days for total perfusion [27,31]. As the main route for the perfusion, the portal vein was used. Alternatively, the hepatic artery or the vena cava served as perfusion route $[14,16,22,26]$. To secure the outflow, the hepatic vessels like the hepatic veins and the vena cava inferior were severed before the perfusion started.

The newest approach was demonstrated in the recently published studies from Struecker et al. $[22,26]$. They introduced perfusion under physiological oscillating pressure conditions via the hepatic artery and the portal vein, and applied this technique successfully in two animal models - rats and pigs.

Other approaches concerning the optimization of the decellularization process were also tested such as the combination of electroporation with simultaneous perfusion [32]. The cryochemical method calls for freezing the freshly explanted liver in distilled water up to $-80^{\circ} \mathrm{C}$ on the 1 st day and slowly thawing on the 2 nd day prior to starting perfusion. This 


\section{European \\ Surgical \\ Research}

\begin{tabular}{l|l}
\hline Eur Surg Res 2016;57:224-239 \\
\hline DOI: 10.1159/000446211 & $\begin{array}{l}\text { ○ 2016 S. Karger AG, Basel } \\
\text { www.karger.com/esr }\end{array}$ \\
\hline
\end{tabular}

Mußbach et al.: Bioengineered Livers: A New Tool for Drug Testing and a Promising Solution to Meet the Growing Demand for Donor Organs

$1 \%$ Triton X-100
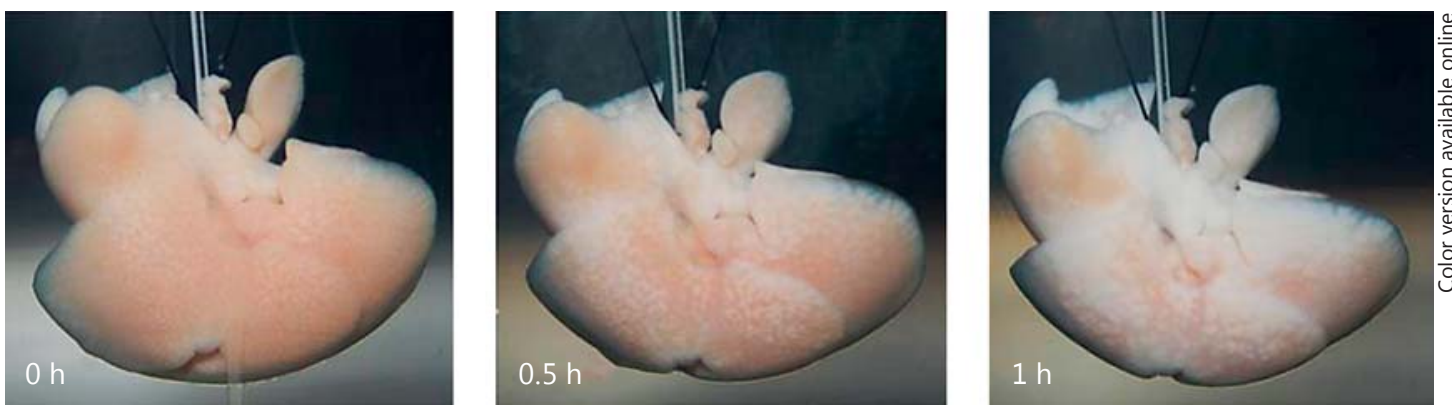

$1 \%$ SDS
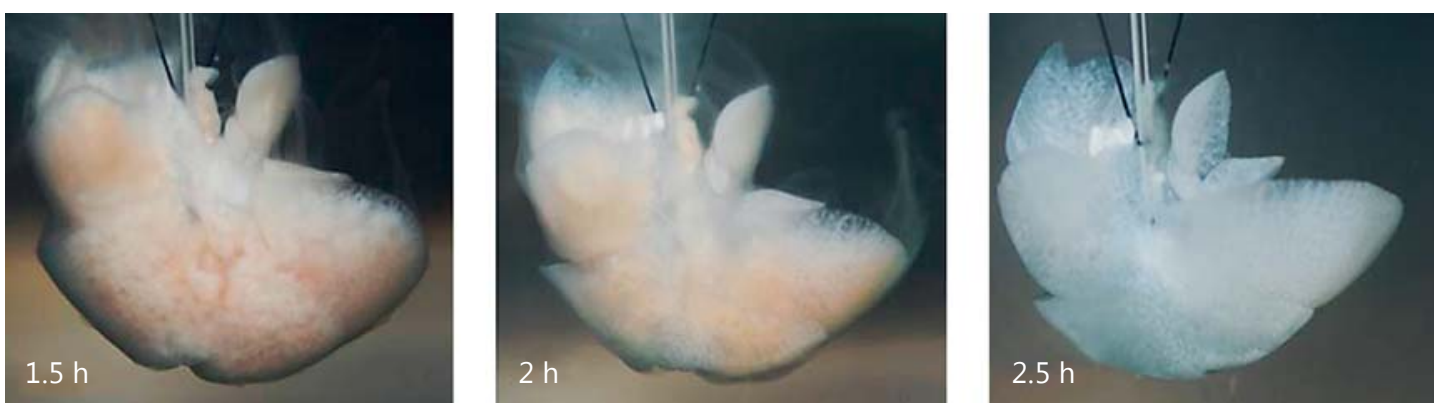

Fig. 1. Decellularization of the liver. After explanting the liver, decellularization was started by perfusing the organ with $1 \%$ Triton X-100 followed by $1 \%$ SDS using a flow rate of $1 \mathrm{ml} / \mathrm{min}$. After approximately $3 \mathrm{~h}$ of perfusion, the scaffold was rinsed with PBS.

freezing procedure leads to the lysis of the cell membranes and damages the intracellular organelles. The subsequent perfusion removes the cellular debris [20].

Few studies aimed to identify the ideal protocol for an effective and gentle decellularization by performing comparative studies applying different protocols $[12,15,21,25,31$, 38]. Comparisons included different solutions to perfuse the organ, mostly SDS and Triton $\mathrm{X}-100$, but also others such as sodium chloride solution, peracetic acid and $1 \%$ NaDOC (Na-deoxycholate), different concentrations of the detergents applied and different routes of perfusion (portal vein and inferior vena cava). However, the unique optimal protocol was not yet identified. All authors reported an almost complete removal of the cellular content from the organs using the different detergents and the maintenance of the components of the extracellular matrix. Triton X-100 constitutes the more gentle option with regard to maintaining the composition of the extracellular matrix and the integrity of the vascular tree. In contrast, using SDS for perfusing the organ scaffold, the decellularization seems to be more effective with more convincing results regarding the removal of cells. Using peracetic acid and the highly concentrated salt solution, a modification of the appearance (for example shrinkage) and ultrastructure of the acellular scaffold was demonstrated [31]. Using different routes of perfusion, no significant difference could be demonstrated regarding the decellularization efficiency. Both options resulted in an acellular organ scaffold with maintained organ structure and vascular microarchitecture [16].

However, up to now, no standard exists of how to optimally assess the quality of the resulting scaffold. Therefore, the selection of read-out parameters differed substantially between the studies, making a direct comparison rather difficult. 


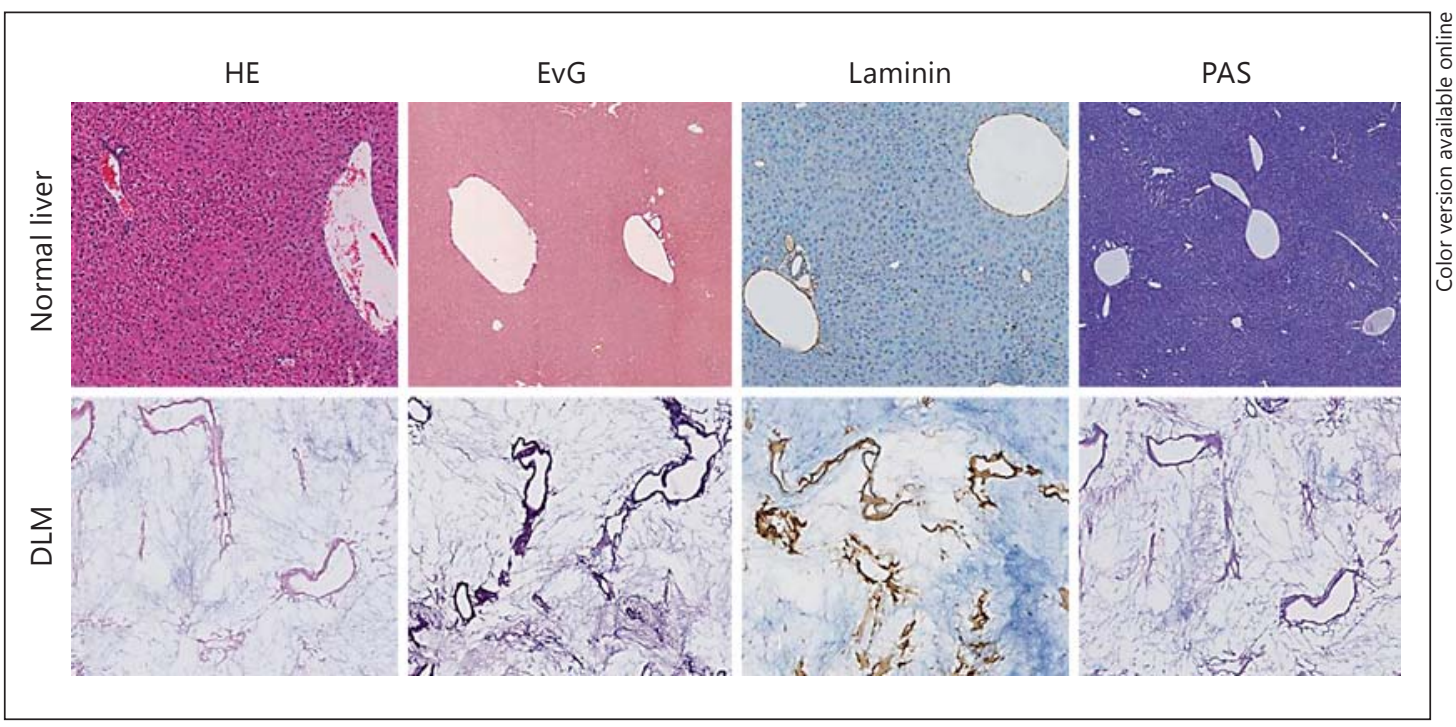

Fig. 2. Histological assessment of the decellularized scaffold. Morphological assessment using HE, elastic Van Gieson (EvG), laminin and PAS staining confirmed the removal of all cells while maintaining the lobular structure and the integrity of the vascular system. DLM = Decellularized liver matrix.

\section{Research Need}

Despite the broad range of studies addressing the decellularization procedure, there are urgent research needs within 3 areas: the selection of the donor species, the impact of ischemia and cardiac arrest, but also organ pathology, on the quality of the organ matrix and the establishment of optimal conditions for decellularization.

In the field of technical optimization, the combined pressure-controlled perfusion seems to be the most promising approach since perfusion was performed under physiological pressure and flow rate conditions. A logical but missing progression aside from the perfusion via the portal vein and hepatic artery is perfusing the biliary tree as well.

\section{Quality Assessment of Decellularization}

A large spectrum of morphological, molecular biological, biochemical and imaging procedures were used in the different studies to assess the success of the decellularization procedure.

\section{Previous Experience}

A common histological method to investigate the complete removal of cells used by many authors is the HE staining (fig. 2). The nuclear staining with DAPI is another frequently used morphological method to investigate the cellular content of the scaffold [13, 29]. A commonly used molecular biological method to determine cell removal is the quantification of the DNA content of the acellular scaffold (fig. 3) [14, 15, 17, 19-21, 27, 29, 39]. Crapo et al. [40] suggested defining a DNA content below $50 \mathrm{ng}$ dsDNA/mg tissue (dry weight) and a fragment length below $200 \mathrm{bp}$ as quality criteria.

The integrity and maintenance of the extracellular matrix are a prerequisite for the successful repopulation, i.e. the survival of the cells after the reseeding procedure, as well as their adhesion and differentiation in the acellular organ scaffold. The composition of the 
Mußbach et al.: Bioengineered Livers: A New Tool for Drug Testing and a Promising

Fig. 3. Molecular assessment of the decellularized scaffold. Decellularization was confirmed by quantifying the DNA content in normal und decellularized liver samples $(n=10)$.

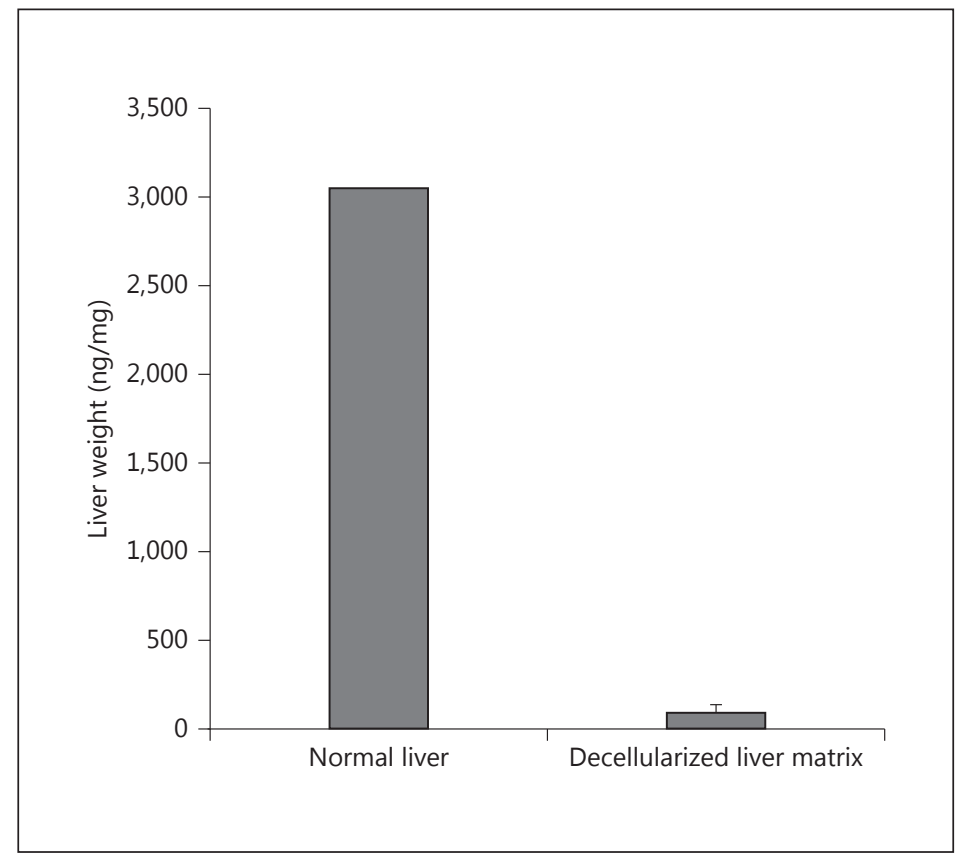

extracellular matrix was investigated by different morphological and biochemical as well as molecular biological methods. To characterize the collagen fibers in the acellular scaffold, Masson trichrome, Russel-Movat pentachrome, Gömöri trichrome, elastic Van Gieson, Sirius red, silver and reticulin staining were applied. The distribution from neutral GAGs (glycosaminoglycans) was demonstrated with PAS and Alcian blue staining. The assessment of specific proteins in the extracellular matrix like collagen I and IV, fibronectin, laminin and elastin was demonstrated with specific immunohistochemical and immunofluorescence staining (fig. 2). The quantitative estimation of the hydroxyproline, collagen, GAG and elastin content permits statements concerning the composition of the extracellular matrix. These morphological estimations were further supported by Western blot data.

The integrity and structure of the vascular tree were assessed by imaging methods. For example CT and MRI with the contrast agents sodium iotalamate and gadopentetate dimeglumine were used to evaluate the integrity of the vascular tree (fig. 4). To confirm the integrity of the vascular structure after perfusion with detergents, a high number of authors employed corrosion casting to visualize the vascular tree $[6,8-10,12,13,16,22,26,29,30]$. Also, first visualizations of the biliary tree were achieved [6, 9, 22, 26, 29].

Different microscopy techniques like scanning electron microscopy (SEM), transmission electron microscopy and fluorescence microscopy were used to visualize the sinusoidal microstructure [7-10, 12-18, 20, 21, 23, 29, 37, 38, 41, 42].

Besides the common and frequently used methods, some authors applied additional methods to demonstrate the integrity of the vascular tree such as the injection of fluorescein bound to dextran via the portal vein, perfusion of trypan blue solution through the vessels and perfusion of the vascular structure with agarose stained with methylene blue $[14,17]$.

\section{Research Need}

Comparing the methods used to decellularize the different organs is difficult because of the widespread spectrum of methods and parameters used to determine the complete removal of the cells and therefore the success of the decellularization. The complete removal of cellular content was confirmed in most studies by HE staining, DAPI staining and DNA 


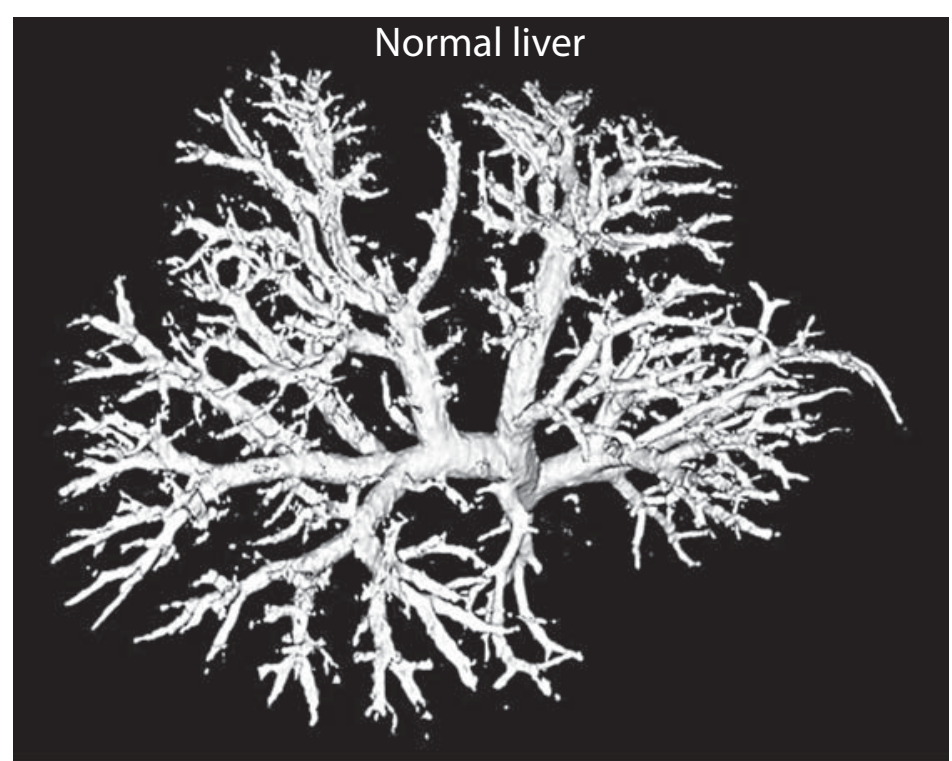

Fig. 4. Assessment of the integrity of the vascular system. Scaffolds were injected with silicone contrast medium and subjected to CT imaging. 3-D reconstruction of the images demonstrated the integrity of the portal venous sys-

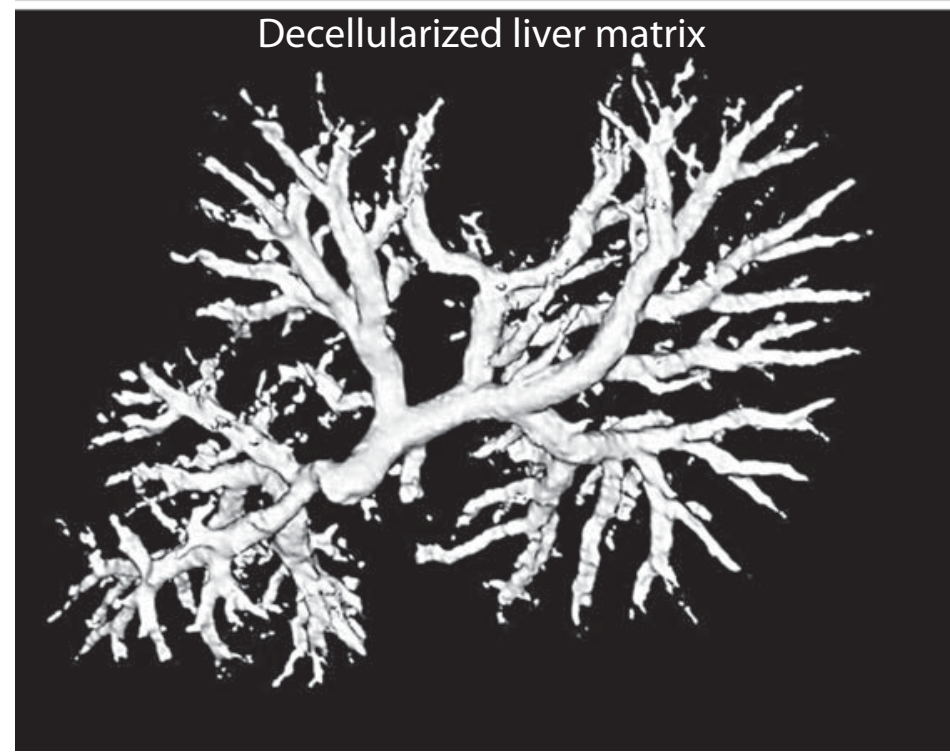
tem.

quantification. In contrast, investigating the composition of the extracellular matrix is far more diverse. Further investigations would benefit from defining a minimal panel of parameters describing the integrity of the extracellular matrix.

Consequently, there is a scientific need to develop a standard protocol to secure a comparable quality assessment of the decellularization procedure especially regarding the composition of the organ scaffold.

\section{Repopulation of the Organ Scaffold}

The repopulation of the organ scaffold is defined as the process to reseed the acellular organ scaffold with organ-specific cells. The aim is to obtain a fully functional organ. Depending on the source of the organ (xenogeneic, allogeneic), the acellular organ scaffold can be reseeded with xenogeneic, allogeneic, isogeneic or autologous cells. 


\begin{tabular}{l|l}
\hline Eur Surg Res 2016;57:224-239 \\
\hline DOI: 10.1159/000446211 & $\begin{array}{l}\text { @ 2016 S. Karger AG, Basel } \\
\text { www.karger.com/esr }\end{array}$ \\
\hline
\end{tabular}

Mußbach et al.: Bioengineered Livers: A New Tool for Drug Testing and a Promising Solution to Meet the Growing Demand for Donor Organs

\section{Previous Experience}

In most studies with small and large animals, the organ scaffolds were reseeded with mature cells, hepatocellular as well as endothelial cell lines and primary hepatocytes with or without simultaneous application of endothelial cells. In the remaining studies, stem cells, mainly mesenchymal stem cells (MSC), were applied for repopulating the organ scaffold.

The majority of the authors used primary hepatocytes to reseed the organ scaffold and to reconstitute the hepatic parenchyma. In many studies, it was demonstrated that the organ scaffold and the retained extracellular matrix components are able to support the growth and survival of the repopulating cells [9, 13, 16-18, 29-31, 37, 38]. Besides repopulating the scaffold with parenchymal cells, reseeding of the vascular tree with endothelial cells is needed and has already been demonstrated [12, 23, 24]. After injection into the vascular system, the cells seemed to engraft into their usual locations and natural niches [12,24].

Another choice is the use of stem and progenitor cells including MSCs, fetal stem cells and hepatic stem cells [7, 20, 30, 37] as well as bone marrow-derived stem cells [4].

First reports revealed that the organ scaffold is able to support the differentiation of these stem cells. Baptista et al. [23] demonstrated the differentiation of fetal hepatoblasts into biliary and hepatic precursor cells by detecting CK-19-positive tubular structures and CK-18 as well as ALB-positive cells in the organ scaffold. MSCs actually showed a higher expression of hepatic markers and an increased metabolic function after the application in the organ scaffold compared to an in vitro culture [20].

Differentiation of stem cells into hepatocytes and endothelial cells prior to reseeding the organ is a promising but time-consuming alternative. The differentiation was mostly achieved in vitro applying defined culture conditions [19]. Stem cells were cultured for 2-4 weeks using a medium supplemented with growth factors [20,37]. Using differentiated stem cells is of advantage since these cells exhibit structural and functional characteristics of mature parenchymal and endothelial cells.

Besides the choice of the optimal source of the cells, the reseeding conditions are crucial to ensure the homogeneous distribution of the cells within the organ scaffold.

Taking the needed high cell number into consideration, dynamic application via a multistep perfusion of the hepatocytes through the vascular system of the organ scaffold was investigated [43]. The dynamic perfusion resulted in a uniform distribution of the reseeded cells within the organ scaffold in contrast to a single injection into the vascular system or the parenchyma of the organ scaffold [6]. The distribution was demonstrated by means of the adhesion of the cells in the organ scaffold and the evaluation via SEM of different areas of the organ scaffold. The measurement via SEM indicated the engraftment of the hepatocytes around the bigger vessels and within the parenchyma [9].

Two routes of application, vena cava and portal vein, were evaluated. Perfusion via vena cava led to a pericentrally focused distribution, whereas perfusion via portal vein resulted in a rather periportal distribution of cells within the organ scaffold. The density of the repopulation was higher when using vena cava and portal vein simultaneously for the dynamic reseeding of the cells [23].

After successful reseeding of the cells, several days of in vitro cultivation of the repopulated organ scaffold are needed. Regarding perfusion conditions, a distinction is made between static and dynamic cultivation. Under static conditions, the reseeded organ is immersed in culture medium, and no further technical devices for perfusing the scaffold are needed. However, in this case, the supply of the repopulated organ scaffold with nutrients and oxygen is limited. Under dynamic conditions, the organ scaffold is continuously perfused with medium using a defined flow rate. This technique allows for a high exchange of oxygen and nutrients, as demonstrated previously. Mirmalek-Sani and colleagues successfully 
Mußbach et al.: Bioengineered Livers: A New Tool for Drug Testing and a Promising

Solution to Meet the Growing Demand for Donor Organs

cultivated the organ scaffold reseeded with the human hepatic cell line HepG2 for 21 days $[12,38]$.

The techniques to assess the repopulation are as manifold as their methods. The success of the repopulation was verified by assessing the density and the homogeneity of the distribution of the reseeded cells in the scaffold. In addition, the success of repopulation was also judged based on the metabolic efficiency of the parenchymal cells and the reendothelialization of the vascular system. HE and DAPI staining was applied to assess cell density and distribution. To verify homogenous distribution, morphological imaging techniques like SEM, transmission electron microscopy and confocal laser scanning microscopy were employed. The microarchitecture of the reseeded organ was mainly explored using HE-stained sections, whereas electron microscopy was used only in a limited number of studies.

Histological and protein-biochemical methods like the detection of different surface proteins such as laminin, elastin, fibronectin and collagens employing immunohistochemistry or immunofluorescence and Western blot were used. Molecular biological methods included DNA quantification and gene expression analysis of differentiation markers and parameters of liver metabolism. Fluorescence microscopy was used to demonstrate that green fluorescent protein-labeled endothelial cells covered the lumen of the vessels and attached to them. However, complete endothelialization could not be shown until now [23].

\section{Research Need}

Regarding the repopulation process, 3 goals need to be achieved to generate a functional organ: (1) reseeding the scaffold with a sufficient amount of parenchymal cells to meet the metabolic needs of the recipient; (2) reendothelialization of the vascular trees to avoid thrombosis while perfusing the scaffold with blood and to ensure the supply of the parenchymal cells with oxygen and nutrients, and (3) appropriate epithelialization of the biliary tree.

To reach these goals, scientific as well as ethical concerns must be considered. The following factors need to be taken into consideration for the process of reseeding the scaffold in vitro: the source of the cells, which is suitable for all intended types of application, the cell types and number of cells for the reseeding, the application conditions like the flow rate, mode of injection (constant/dynamic), one step or multistep application, and application route (application via the portal vein or hepatic artery) as well as the optimization of the subsequent culture conditions of the reseeded organ scaffold.

The immense diversity of the published techniques indicates the lack of an ideal approach for assessing the quality of repopulation. A comparative quality assessment is currently virtually impossible due to the variety of methods and parameters used to demonstrate the complete repopulation of the organ scaffold. Up to now, merely a few studies investigated the integrity of the biliary tract after repopulation, a topic also deserving attention. Further effort must be made to develop a method for evaluating the reseeding of the biliary tract and to standardize the evaluation algorithm. Last but not least, the 3-D microarchitecture of the reseeded scaffold needs to be explored in more detail.

\section{Functional Assessment of the Repopulated Liver}

Up to now, there is only limited experience in evaluating the functional capacity of the repopulated liver organ. Functional assessment was performed under all conditions: using scaffolds from small and large animals, repopulated with mature as well as with stem cells and cultured under static as well as under perfusion conditions. Functional assessment of the repopulated liver was mainly limited to quantification of basic metabolic parameters such as 
Mußbach et al.: Bioengineered Livers: A New Tool for Drug Testing and a Promising

Solution to Meet the Growing Demand for Donor Organs

albumin secretion, urea production, bile acid production and ammonia elimination $[6,8,13$, $16,18-20,29-31]$. In some cases, drug metabolism was investigated by assessing the CYP system $[7,15,23,30,42]$.

\section{Previous Experience}

Culturing cells in the natural environment of the scaffold after reseeding seemed to result in higher metabolic activity compared to conventional culturing systems, irrespective of the source and type of cells. A higher metabolic activity was observed when using primary hepatocytes (rat, pig) for reseeding the scaffold and applying perfusion culturing condition compared to the static culturing condition using collagen-coated culture dishes. As key parameters, albumin secretion and urea synthesis were used as well as ammonia elimination and the relative mRNA expression of CYP proteins $[15,29]$.

When using bone marrow-derived MSCs from Balb/c mice for repopulating the scaffold, differentiation into hepatocytes was observed after 4 weeks of perfusion culture. This finding was confirmed by immunostaining (PAS, albumin, AFP, CK-19) using normal mouse liver sections as positive control. The metabolic activity in terms of albumin secretion and urea production of the MSCs repopulating the scaffold was higher compared to a 2-D culture [20].

Using fetal human liver cells to reseed a porcine acellular scaffold resulted in the maturation of the cells as demonstrated by immunostaining of different hepatic markers and a superior metabolic function in terms of urea and albumin production compared to fetal liver cells in 2-D [23].

Using human hepatic stem cells for reseeding an acellular rat scaffold also resulted in maturation as indicated by immunohistochemistry, RT-PCR and superior hepatic function in terms of urea synthesis and CYP3A4 activity compared to a 2-D culture [7].

\section{Research Need}

A standardized functional assessment of the reseeded biological scaffold is needed. The function of the reseeded cells should be investigated in comparison to freshly isolated hepatocytes cultured under optimal conditions. The assessment should include basic parameters such as the elimination of ammonia, urea synthesis, bile acid and albumin production and secretion.

The need for assessing additional parameters such as drug metabolism proteins and liver enzymes is another issue which should be addressed. Comparative gene expression studies using gene arrays (e.g. Affymetrix) would contribute to further characterization of the functional quality of the reseeded scaffold. Both functional assessment and gene expression studies will contribute to a comprehensive characterization prior to further use in pharmacological research and transplantation studies.

\section{Use of Repopulated Organs as Human Cell Culture Systems}

The studies focusing on the use of the repopulated scaffolds as humanized cell culture differ in terms of the cell type used for repopulation and in terms of nutrient supply.

\section{Previous Experience}

Several types of human cells were used for repopulation in the context of a humanized cell culture system. Two different culture approaches were investigated: nutrient supply via diffusion using repopulated liver sections and nutrient supply via perfusion using rodent livers or liver lobes from large animals. 
Discs from the acellular scaffold obtained from perfused porcine livers were seeded with the human hepatic cell line HepG2 and maintained under static culture conditions. Using cell proliferation assays, the optimized disc was selected and seeded in a second step with human hepatocytes, and the latter were cultivated for 21 days. Cultivating the hepatocytes on these extracellular matrix discs resulted in considerably improved cell growth and sustained cell function. In summary, it was concluded that these liver scaffolds 'better represent the natural in-vivo environment in an ex-vivo system' [31].

As an alternative, human hepatic stem cells were seeded onto small cryosections of the acellular scaffold of a rat liver. These cells were placed onto a 24-well cell culture plate and cultivated for 8 weeks. The stem cells lost their stem cell markers and differentiated into mature, functional parenchymal cells within 1 week. Cells could be maintained viable for as long as 8 weeks under theses culture conditions. These results indicate that the scaffolds were suitable for inducing the differentiation of stem cells into mature and functional cells. These functional organ sections could then be used for clinical and industrial purposes [7].

In 2009, the first study postulating the use of repopulated scaffolds as perfusion cell culture system was performed. HepG2 cells were infused via the portal vein into a decellularized ferret liver scaffold, and the viability and growth of the reseeded cells throughout the 7-day observation period was demonstrated. Based on these results, the authors concluded that repopulated scaffolds may serve as 'new drug discovery platforms and most needed organs for transplantation' [24].

Small cubes from human scaffolds were reseeded with 3 types of human liver cells (LX2 hepatic stellate cells, Sk-Hep-1 from hepatocellular carcinoma and a hepatoblastoma cell line HepG2) and cultured for 3, 7 and 21 days under static conditions. The increasing cell number was taken as one parameter of a successful repopulation [40].

Complexity was increased when using two different human cells for repopulation of the ferret scaffold. A coculture of umbilical vein endothelial cells and fetal liver cells resulted in the successful repopulation of the scaffold. A 10-fold increase in the DNA concentration after a cultivation period of 7 days and HE staining showed dense cellularity throughout the whole scaffold. The proliferation of the cells was shown by immunofluorescence staining of Ki67. The cells engrafted in their native locations within the acellular organ scaffold. Another promising result was that these cells displayed typical endothelial, hepatic and biliary epithelial markers. Therefore, Baptista et al. [23] suggested that the repopulated scaffolds are able to create a liver-like tissue in vitro.

\section{Research Need}

The studies demonstrated the suitability of decellularized liver scaffolds to support the growth of reseeded human cells. The use of repopulated functional xenogeneic organ scaffolds for humanized cell culture systems for pharmacological studies is tangible in the foreseeable future. A prerequisite for this application is the identification of the optimal human cell type to fully repopulate the organ. These cells need to be able to demonstrate a fully functional drug metabolism system as well as a long survival and/or proliferation even after a long cultivation period.

\section{Use of Repopulated Organs for Transplantation}

The ultimate goal of organ engineering is the therapy of end-stage organ failure by the transplantation of the repopulated organ. Different experimental approaches were pursued - transplantation of the acellular scaffold to test biocompatibility and immunogenicity 
Mußbach et al.: Bioengineered Livers: A New Tool for Drug Testing and a Promising

Solution to Meet the Growing Demand for Donor Organs

and in vivo repopulation as well as transplantation of reseeded liver tissue, and auxiliary transplantation of individual lobes. Orthotopic transplantation of a repopulated full liver graft has yet to be performed.

\section{Previous Experience}

To explore biocompatibility, heterotopic transplantation of acellular scaffolds was performed. Transplantation of decellularized scaffolds into living animals of the same or a different species did not result in a major inflammatory response. This was demonstrated by comparing the inflammatory response after transplanting either an allogeneic rat liver scaffold or a xenogeneic porcine scaffold into rat subcutaneous fat tissue. In both cases, repopulation with host cells was demonstrated, but no infiltration with inflammatory cells. Systemic inflammatory parameters were also low in both groups [30]. Similar results were obtained in another study where small acellular tissue blocks from rat livers were transplanted in the back muscle of rabbits. Systemic testing for eventual elevation of serum CRP or serum IgE levels did not indicate the initiation of an inflammatory response. Additionally, the histology showed 'no obvious infiltration' with inflammatory cells. Further confirmation was achieved when transplanting cubic pieces of the decellularized human scaffolds into immunocompetent mice. The transplanted tissue cubes showed 'no signs of inflammation or adverse tissue reactions around the implants' [16].

To explore in vivo repopulation of tissue cubes, different heterotopic implantation sites were tested such as the subcutaneous area and the omentum. After a period of 7 and 21 days, the implants were harvested and evaluated by histology or immunohistochemistry. The alpha smooth muscle actin staining indicated on the one hand the infiltration of fibroblasts into the implants and on the other hand an 'evidence for neovascularization of the implant'. HE and CD3 staining indicated a lack of severe inflammation reaction and infiltrated inflammatory cells [33].

Small pieces of acellular livers from rats and pigs were implanted in the subhepatic area of recipient rats and were fixed to the right liver lobe with 4 simple sutures without revascularization. Repopulation with binuclear hepatocyte-like cells was demonstrated by hepatocyte staining with more distinct results in the allogenic model than the xenogenic model. In both cases, only a minor inflammatory reaction with infiltration of inflammatory cells was shown by HE staining [21].

Transplantation of in vitro repopulated scaffolds was also investigated. In a mouse model with hepatic failure after administration of $\mathrm{CCl}_{4}$, little pieces of acellular scaffolds as well as reseeded scaffolds after repopulation with MSCs functioned as grafts. The grafts were implanted directly into the hepatic parenchyma and were secured by hemostatic mesh and the application of biological glue. After the transplantation of the reseeded scaffolds, an improvement in liver functions could be demonstrated, and the animals fully recovered after 7 days. The histological examinations indicated an engraftment of the reseeded organ scaffold into the region of the lobule. Also, the highest survival rate was reached in the experimental group which received a transplantation of the reseeded organ construct [20].

Small pieces of reseeded organ constructs were transplanted as auxiliary grafts in the left renal space of the recipient rat after a partial nephrectomy. To revascularize the graft, the renal artery of the recipient rat was anastomosed with the portal vein of the scaffold. Drainage was established by anastomosing the scaffold's inferior vena cava with the renal vein of the recipient rat. After 8-hour observation time, the graft was explanted for further examinations (HE, albumin, glucose-6-phosphate, Ugt1a). Using the TUNEL assay, an apoptosis rate of $20 \%$ was detected reflecting the hepatocellular damage during the 8-hour observation time. The histological/immunohistochemical examinations confirmed the morphology and the parenchymal position of the hepatocytes as well as the retained hepatic function in the transplanted grafts [6]. 
Mußbach et al.: Bioengineered Livers: A New Tool for Drug Testing and a Promising Solution to Meet the Growing Demand for Donor Organs

The transplantation of the reseeded right median liver lobe after a $90 \%$ partial hepatectomy prolonged the survival time of rats from 16 to $72 \mathrm{~h}$. Additionally, gene expression analysis of the scaffolds after this 72-hour transplantation period revealed that the hepatocytes applied for the reseeding express liver-related genes. It was demonstrated that the hepatocytes express coagulation factor X (F10), albumin, cytochrome P450 (CYP1a2), acetoacetyl-CoA synthetase, triacyl-glycerol hydrolase and the transcription factor PPAR $\alpha$, which is involved in lipid oxidation. Furthermore, the reseeded scaffold was able to synthesize albumin, and the PAS staining demonstrated the presence of glycogen [42].

\section{Research Need}

The 'ideal' animal model to test the efficiency of the whole liver engineering process remains the orthotopic transplantation of a reseeded complete organ. Despite all reports describing the success of repopulating a decellularized organ scaffold, none of the published articles explored the viability and functionality of the repopulated liver construct after orthotopic transplantation. In all reports, the observation time was limited to several days only.

For transplantation, three challenges need to be met: (1) the development of a suitable surgical model for orthotopic, potentially auxiliary, transplantation of the repopulated liver scaffolds; (2) the demonstration of the successful life-sustaining effect of transplanting a repopulated whole organ in small animals, and (3) subsequently in large animals. Solving these problems is the absolute prerequisite prior to attempting a clinical transplantation.

\section{Summary, Achievements and Research Needs}

In summary, generating functional biological organs by liver engineering represents a novel strategy to potentially alleviate the lack of organs suitable for both applications: (1) as humanized cell culture system in the pharmacological research for extensive drug testing and (2) as surrogates/replacements for transplantation to treat organ failure. Liver decellularization and repopulation is principally possible; however, long-term success has not been reported yet.

Further optimization and standardization are needed to identify the optimal protocol for decellularization and quality control. According to current publications and our own experience, pulsatile perfusion with SDS and Triton X-100 using portal vein and hepatic artery is the most efficient approach. More research is needed to identify the best protocol for quality control of the scaffold. This should include a qualitative and quantitative assessment of the matrix protein composition.

A tremendous need for further research exists in the field of repopulation and functional assessment of the repopulated organ scaffold in vitro/in vivo and the establishment of transplantation models. Optimization of repopulation protocols based on repeated reseeding via portal vein and hepatic artery and on culturing under perfusion conditions deserve further attention. One of the most difficult issues is the determination of the standard panel of parameters for quality control of the repopulated scaffold prior to implantation. The ultimate functional criteria should be graft survival time without hepatic dysfunction and without architectural impairment after orthotopic transplantation of the repopulated scaffold.

Prior to optimizing protocols, principal decisions have to be made regarding the selection of the source of organs for decellularization and the source of cells for repopulation. Basic research should be restricted to rats and mice. For preclinical research, the use of porcine and nontransplantable human organs is most appealing. Repopulation of the scaffolds with MSCs from the future recipient seems to be the most promising approach. In this case, only one cell 
Mußbach et al.: Bioengineered Livers: A New Tool for Drug Testing and a Promising

Solution to Meet the Growing Demand for Donor Organs

type would be needed for the ex vivo repopulation and reconstitution of hepatic parenchyma as well as the vascular and biliary tree. Furthermore, the need for immunosuppression after transplantation of the newly engineered organ graft would be eliminated.

Solving these issues will be a prerequisite for the application of bioengineered livers in pharmacological research and for a contribution to reduce organ shortage.

\section{Acknowledgement}

The authors sincerely thank Isabel Jank for language editing and language revision and Stephanie Lange, Bianca Göhrig, Kathrin Schulze and Elke Oswald for their technical assistance and expert animal care.

\section{Statement of Ethics}

The published work complies with the guidelines for human and animal welfare regulations. The animal experiments conform to institutional standards and are approved by the corresponding ethical committee on animal research (Reg. No. 02-074/14).

\section{Disclosure Statement}

Franziska Mußbach, Utz Settmacher, Olaf Dirsch, Chichi Xie and Uta Dahmen declare that they have no conflict of interest.

\section{References}

1 Murphy SV, Atala A: Organ engineering - combining stem cells, biomaterials, and bioreactors to produce bioengineered organs for transplantation. Bioessays 2013;35:163-172.

2 Ott HC, Matthiesen TS, Goh SK, et al: Perfusion-decellularized matrix: using nature's platform to engineer a bioartificial heart. Nat Med 2008;14:213-221.

3 Khan AA, Vishwakarma SK, Bardia A, et al: Repopulation of decellularized whole organ scaffold using stem cells: an emerging technology for the development of neo-organ. J Artif Organs 2014;17:291-300.

4 Scarritt ME, Pashos NC, Bunnell BA: A review of cellularization strategies for tissue engineering of whole organs. Front Bioeng Biotechnol 2015;3:43.

5 Shupe T, Williams M, Brown A, et al: Method for the decellularization of intact rat liver. Organogenesis 2010; 6:134-136.

6 Uygun BE, Soto-Gutierrez A, Yagi H, et al: Organ reengineering through development of a transplantable recellularized liver graft using decellularized liver matrix. Nat Med 2010;16:814-820.

7 Wang Y, Cui CB, Yamauchi M, et al: Lineage restriction of human hepatic stem cells to mature fates is made efficient by tissue-specific biomatrix scaffolds. Hepatology 2011;53:293-305.

8 Uygun BE, Price G, Saedi N, et al: Decellularization and recellularization of whole livers. J Vis Exp 2011;48:pii 2394.

9 Soto-Gutierrez A, Zhang L, Medberry C, et al: A whole-organ regenerative medicine approach for liver replacement. Tissue Eng Part C Methods 2011;17:677-686.

10 De Kock J, Ceelen L, De Spiegelaere W, et al: Simple and quick method for whole-liver decellularization: a novel in vitro three-dimensional bioengineering tool? Arch Toxicol 2011;85:607-612.

11 Park KM, Woo HM: Systemic decellularization for multi-organ scaffolds in rats. Transplant Proc 2012;44: 1151-1154.

12 Shirakigawa N, Ijima H, Takei T: Decellularized liver as a practical scaffold with a vascular network template for liver tissue engineering. J Biosci Bioeng 2012;114:546-551.

13 Cheng Y, Wang Y, Kang YZ, et al: In vitro culture of tumour-derived hepatocytes in decellularised whole-liver biological scaffolds. Digestion 2013;87:189-195.

14 Nari GA, Cid M, Comin R, et al: Preparation of a three-dimensional extracellular matrix by decellularization of rabbit livers. Rev Esp Enferm Dig 2013;105:138-143.

15 Ren H, Shi X, Tao L, et al: Evaluation of two decellularization methods in the development of a whole-organ decellularized rat liver scaffold. Liver Int 2013;33:448-458.

16 Pan MX, Hu PY, Cheng Y, et al: An efficient method for decellularization of the rat liver. J Formos Med Assoc 2014;113:680-687. 
17 Hussein KH, Park KM, Teotia PK, et al: Fabrication of a biodegradable xenoantigen-free rat liver scaffold for potential drug screening applications. Transplant Proc 2013;45:3092-3096.

18 Gessner RC, Hanson AD, Feingold S, et al: Functional ultrasound imaging for assessment of extracellular matrix scaffolds used for liver organoid formation. Biomaterials 2013;34:9341-9351.

19 Lee JS, Shin J, Park HM, et al: Liver extracellular matrix providing dual functions of two-dimensional substrate coating and three-dimensional injectable hydrogel platform for liver tissue engineering. Biomacromolecules 2014;15:206-218.

20 Jiang WC, Cheng YH, Yen MH, et al: Cryo-chemical decellularization of the whole liver for mesenchymal stem cells-based functional hepatic tissue engineering. Biomaterials 2014;35:3607-3617.

21 Sabetkish S, Kajbafzadeh AM, Sabetkish N, et al: Whole-organ tissue engineering: decellularization and recellularization of three-dimensional matrix liver scaffolds. J Biomed Mater Res A 2015;103:1498-1508.

22 Struecker B, Butter A, Hillebrandt K, et al: Improved rat liver decellularization by arterial perfusion under oscillating pressure conditions. J Tissue Eng Regen Med 2014, Epub ahead of print.

23 Baptista PM, Siddiqui MM, Lozier G, et al: The use of whole organ decellularization for the generation of a vascularized liver organoid. Hepatology 2011;53:604-617.

24 Baptista PM, Orlando G, Mirmalek-Sani SH, et al: Whole organ decellularization - a tool for bioscaffold fabrication and organ bioengineering. Conf Proc IEEE Eng Med Biol Soc 2009;2009:6526-6529.

25 Wang Y, Bao J, Wu Q, et al: Method for perfusion decellularization of porcine whole liver and kidney for use as a scaffold for clinical-scale bioengineering engrafts. Xenotransplantation 2015;22:48-61.

26 Struecker B, Hillebrandt KH, Voitl R, et al: Porcine liver decellularization under oscillating pressure conditions - a technical refinement to improve the homogeneity of the decellularization process. Tissue Eng Part $\mathrm{C}$ Methods 2015;21:303-313.

27 Mattei G, Di PV, Tirella A, et al: Mechanostructure and composition of highly reproducible decellularized liver matrices. Acta Biomater 2014;10:875-882.

28 Kajbafzadeh AM, Javan-Farazmand N, Monajemzadeh M, et al: Determining the optimal decellularization and sterilization protocol for preparing a tissue scaffold of a human-sized liver tissue. Tissue Eng Part C Methods 2013;19:642-651.

29 Yagi H, Fukumitsu K, Fukuda K, et al: Human-scale whole-organ bioengineering for liver transplantation: a regenerative medicine approach. Cell Transplant 2013;22:231-242.

30 Barakat 0 , Abbasi S, Rodriguez G, et al: Use of decellularized porcine liver for engineering humanized liver organ. J Surg Res 2012;173:e11-e25.

31 Lang R, Stern MM, Smith L, et al: Three-dimensional culture of hepatocytes on porcine liver tissue-derived extracellular matrix. Biomaterials 2011;32:7042-7052.

32 Sano MB, Neal RE, Garcia PA, et al: Towards the creation of decellularized organ constructs using irreversible electroporation and active mechanical perfusion. Biomed Eng Online 2010;9:83.

33 Mazza G, Rombouts K, Rennie Hall A, et al: Decellularized human liver as a natural 3D-scaffold for liver bioengineering and transplantation. Sci Rep 2015;5:13079.

34 Deutsche Stiftung Organtransplantation: Organspende und Transplantation in Deutschland. Jahresbericht 2014. Frankfurt am Main, Deutsche Stiftung Organtransplantation, 2015.

35 Badylak SF, Taylor D, Uygun K: Whole-organ tissue engineering: decellularization and recellularization of three-dimensional matrix scaffolds. Annu Rev Biomed Eng 2011;13:27-53.

36 Kang YZ, Wang Y, Gao Y: Decellularization technology application in whole liver reconstruct biological scaffold (in Chinese). Zhonghua Yi Xue Za Zhi 2009;89:1135-1138.

37 He H, Liu X, Peng L, et al: Promotion of hepatic differentiation of bone marrow mesenchymal stem cells on decellularized cell-deposited extracellular matrix. Biomed Res Int 2013;2013:406871.

38 Mirmalek-Sani SH, Sullivan DC, Zimmerman C, et al: Immunogenicity of decellularized porcine liver for bioengineered hepatic tissue. Am J Pathol 2013;183:558-565.

39 Uygun BE, Yarmush ML, Uygun K: Application of whole-organ tissue engineering in hepatology. Nat Rev Gastroenterol Hepatol 2012;9:738-744.

40 Crapo PM, Gilbert TW, Badylak SF: An overview of tissue and whole organ decellularization processes. Biomaterials 2011;32:3233-3243.

41 Ye JS, Stoltz JF, de Isla N, et al: An approach to preparing decellularized whole liver organ scaffold in rat. Biomed Mater Eng 2015;25:159-166.

42 Bao J, Shi Y, Sun H, et al: Construction of a portal implantable functional tissue-engineered liver using perfusiondecellularized matrix and hepatocytes in rats. Cell Transplant 2011;20:753-766.

43 Zhou Q, Li L, Li J: Stem cells with decellularized liver scaffolds in liver regeneration and their potential clinical applications. Liver Int 2015;35:687-694. 